\title{
Wind and nebula of the M 33 variable GR 290 (WR/LBV) ${ }^{\star}$
}

\author{
Olga Maryeva ${ }^{1}$, Gloria Koenigsberger ${ }^{2}$, Oleg Egorov ${ }^{3}$, Corinne Rossi ${ }^{4}$, Vito Francesco Polcaro ${ }^{5, \star \star}$, \\ Massimo Calabresi ${ }^{6}$, and Roberto F. Viotti ${ }^{5}$
}

\author{
1 Astronomical Institute, Czech Academy of Sciences, Fričova 298, 25165 Ondřejov, Czech Republic \\ e-mail: olga.maryeva@asu.cas.cz \\ 2 Instituto de Ciencias Físicas, Universidad Nacional Autónoma de México, Ave. Universidad s/n, 62210 Cuernavaca, Morelos, \\ México \\ e-mail: gloria@astro.unam.mx \\ ${ }^{3}$ Lomonosov Moscow State University, Sternberg Astronomical Institute, Universitetsky pr. 13, 119234 Moscow, Russia \\ e-mail: egorov@sai.msu.ru \\ ${ }^{4}$ Università Sapienza, Piazza A. Moro 5, 00185 Roma, Italy \\ 5 INAF-Istituto di Astrofisica e Planetologia Spaziali di Roma (IAPS-INAF), Via del Fosso del Cavaliere 100, 00133 Roma, Italy \\ ${ }^{6}$ Associazione Romana Astrofili, Roma, Italy
}

Received 22 December 2017 / Accepted 29 May 2018

\begin{abstract}
Context. GR 290 (M33/V532 = Romano's Star) is a suspected post-luminous blue variable star located in M 33 galaxy that shows a rare Wolf-Rayet (WR) spectrum during its minimum light phase. In spite of many studies, its atmospheric structure, its circumstellar environment, and its place in the general context of massive stars' evolution is poorly known.

Aims. We present a detailed study of this star's wind and mass loss, and a study of the circumstellar environment associated to the star.

Methods. Long-slit spectra of GR 290 were obtained during its present minimum luminosity phase with the Gran Telescopio Canarias covering the $\sim 3600-7500 \AA$ wavelength range together with contemporaneous photometry using $B, V, R$ and $I$ filters. The data were compared with non-local thermodynamical equilibrium (non-LTE) model atmosphere synthetic spectra computed with CMFGEN code and with models for ionized interstellar medium regions computed with CLOUDY code.

Results. The current $m_{\mathrm{V}}=18.8 \mathrm{mag}$ is the faintest at which this source has ever been observed. The non-LTE models indicate effective temperatures of $T_{\text {eff }}=27000-30000 \mathrm{~K}$ at radius $R_{2 / 3}=27-21 R_{\odot}$ and mass-loss rate $\dot{M}=1.5 \times 10^{-5} M_{\odot} \mathrm{yr}^{-1}$. The terminal wind speed $v_{\infty}=620 \mathrm{~km} \mathrm{~s}^{-1}$ is faster than ever before recorded, while the current luminosity $L_{*}=(3.1-3.7) \times 10^{5} L_{\odot}$ is the lowest ever deduced. The star is overabundant in $\mathrm{He}$ and $\mathrm{N}$ and underabundant in $\mathrm{C}$ and $\mathrm{O}$. It is surrounded by an unresolved compact $\mathrm{H}$ II region with dimensions $\leq 4 \mathrm{pc}$, from where $\mathrm{H}$-Balmer, He I lines, and $[O I I I]$ and $[N I I]$ are detected. In addition, we find emission from a more extended interstellar medium (ISM) region, which appears to be asymmetric, with a larger extent to the east (16-40 pc) than to the west.

Conclusions. In the present long lasting visual minimum, GR 290 is in a lower bolometric luminosity state with higher mass-loss rate. The nearby nebular emission seems to suggest that the star has undergone significant mass loss over the past $10^{4}-10^{5} \mathrm{yr}$ and is nearing the end stages of its evolution.
\end{abstract}

Key words. stars: Wolf-Rayet - stars: variables: S Doradus - stars: winds, outflows - stars: evolution - stars: individual: GR 290 galaxies: individual: M33

\section{Introduction}

Luminous blue variables (LBVs) are a relatively short evolutionary stage in the life of massive stars. During this phase, stars lose significant mass through strong stellar winds and occasional giant eruptive events. As a result, they shed their outer layers and eventually become hydrogen-deficient Wolf-Rayet (WR) stars (Langer et al. 1994; Humphreys \& Davidson 1994; Ekström et al. 2012; Groh et al. 2013b).

* Based on observations made with the Gran Telescopio Canarias (GTC), installed at the Spanish Observatorio del Roque de los Muchachos of the Instituto de Astrofísica de Canarias, in the island of $\mathrm{La}$ Palma and with the Cassini 1.52-m telescope of the Bologna Observatory (Italy)

$\star \star$ Deceased on February 11, 2018.
LBVs show both short timescale stochastic variability with amplitude up to a few tenths of magnitude (Humphreys \& Davidson 1994; Abolmasov 2011) and long-term variability, socalled S Dor cycle (Humphreys \& Davidson 1994; Vink 2012). Typically S Dor cycles last for several years and their amplitude is 1-2 mags (Humphreys et al. 2016). During S Dor cycles, photometric variability is always accompanied by changes in effective temperature, that is, spectral type of a star changes in the range from $\mathrm{B}$ supergiants or late $\mathrm{Of} / \mathrm{WN}$ stars to A-supergiants. At the same time, the bolometric luminosity remains approximately constant (Wolf 1989; Humphreys \& Davidson 1994) or may even decrease during the visual maximum (Groh et al. 2009). Moreover, Galactic objects $\eta$ Car and P Cyg have shown another kind of outbursts, so-called giant eruptions; these are a huge increase of bolometric luminosity simultaneously with an increase of visual brightness (Humphreys \& Davidson 1994). 
LBVs are divided into two subclasses: low luminosity LBVs and classical LBVs (Humphreys et al. 2016). The former originate from stars with $M_{*} \approx 25-40 M_{\odot}$ reaching the LBV stage after being red supergiants (RSG; Humphreys et al. 2016; Meynet \& Maeder 2005). Groh et al. (2013a) demonstrated that low luminosity LBVs could be progenitors of supernovae (SN) IIb. Thus low luminosity LBVs are a final stage of stellar evolution, rather than a transitory evolutionary phase between RSG and WR as was suggested earlier (Meynet \& Maeder 2005).

Classical LBVs originate from more massive stars $\left(M_{*} \approx 40-\right.$ $\left.60 M_{\odot}\right)$ moving off the main sequence (MS) and evolving towards the WR stage via the blue supergiant (BSG) and LBV phases (Meynet \& Maeder 2005; Meynet et al. 2011). According to Groh et al. (2014), stars with $60 M_{\odot}$ spend $2 \times 10^{5} \mathrm{yr}$ in the LBV phase, only $5 \%$ of their lifetime. Consideration of the initial mass function (IMF) leads us to conclude that LBV stars are extremely rare and there are probably no more than a few dozen such objects in the Galaxy. Clues to the mechanisms by which transition from O-type stars and WR stars occurs may be gleaned from a handful of objects that have been observed to display both WR and LBV characteristics. Examples of these rare objects include AG Car in our Galaxy (Groh et al. 2009), R 127 and HDE 269582 in the Large Magellanic Cloud (Walborn et al. 2017, and references therein), HD 5980 in the Small Magellanic Cloud (Koenigsberger et al. 2014) and GR 290 in M 33 (Polcaro et al. 2016, and references therein).

GR 290 (M33-V532= Romano's Star) is located in a relatively empty field on the outskirts of the M33 galaxy. It was first classified as a Hubble-Sandage variable by Romano (1978) and then became an LBV candidate (Humphreys \& Davidson 1994; Szeifert 1996) after the introduction of this class of objects by Peter Conti (1984). Spectral variations from a B-supergiant type spectrum to that of a WR of the nitrogen sequence $(\mathrm{WN})^{1}$, photometric variability (Kurtev et al. 2001), as well as a large bolometric luminosity (Polcaro et al. 2003) are the reasons that led to the promotion of GR 290 from a LBV candidate to a bona fide LBV (see Kurtev et al. 2001; Polcaro et al. 2003 and Fabrika et al. 2005). Later, Polcaro et al. (2011) concluded that GR 290's bolometric luminosity has significantly changed during the minimum phase of the year 2008 and suggested that Romano's star has already passed through the LBV phase and therefore that it is a post-LBV. Humphreys et al. (2014) noted that the star does not exhibit S Dor transitions to the cool, dense wind state, instead it varies between two hot states on the Hertzsprung-Russell diagram (HRD), characterized by WN spectroscopic features (Humphreys et al. 2014). Humphreys et al. (2014) also, like Polcaro et al. (2011) earlier, suggested that Romano's star is likely in a post-LBV state.

The photometric investigation initiated by Romano (1978) was followed by that of Kurtev et al. (2001), who reported the photometry obtained over 1982-1990. They concluded that the eruption timescales are $\sim 20 \mathrm{yr}$ and that shorter timescale oscillations of $\sim 270$ days or $\sim 320$ days with amplitudes of $0.5 \mathrm{mag}$ are also present. Zharova et al. (2011) investigated the

\footnotetext{
1 There is only one spectrum of GR 290 obtained in 1992 during a major eruption of 1992-1994 and published by Szeifert (1996). This spectrum may be classified as that of a late type supergiant. We may assume that GR 290 has displayed a WN type spectrum since 1994, as all known spectra acquired after that time, starting with the one published by Sholukhova et al. (1997), are of WN type.
}

photometric variability of GR 290 using both the Moscow collection of photographic plates, their own data, and data published earlier (Romano 1978; Humphreys \& Sandage 1980; Sholukhova et al. 2002; Viotti et al. 2006), thus constructing the most comprehensive light curve covering half a century. This light curve shows that GR 290 exhibits irregular brightness variations with different amplitudes and timescales, and where large amplitude and complex wave-like variations on the scale of several years are the most prominent. Polcaro et al. (2016) were able to further extend this light curve to the beginning of the twentieth century and demonstrated that GR 290 did not display any significant eruptions between 1901 and the 1960s.

Studies of GR 290 devoted to spectral variability show that its spectral type changes between WN11 and WN8 (Maryeva \& Abolmasov; Polcaro et al. 2011; Sholukhova et al. 2011). Since the beginning of the $2000 \mathrm{~s}$ it has made this transition twice (Polcaro et al. 2016). Viotti et al. (2006, 2007) first described an anti-correlation between emission line strength and brightness. Then Maryeva \& Abolmasov (2010) found a correlation of spectral changes and the visual brightness typical for LBVs: the brighter it is, the cooler the spectral type. However, among all known LBVs only HD 5980 convincingly shows a hotter spectrum in the minimum of brightness.

The models of GR 290's atmosphere have been constructed for three states: luminosity maximum of 2005, the minimum of brightness in 2008 (Maryeva \& Abolmasov 2012), and at the moderate luminosity maximum of 2010 (Clark et al. 2012). Polcaro et al. (2016) built nine models for the most representative spectra acquired between 2002 and 2014 and demonstrated how the GR 290 wind structure varies correlated with brightness changes; the slow and dense wind at brightness maxima becomes faster and thinner at minima. Similar correlations appear also in HD 5980 (Georgiev et al. 2011) and provide clues to the mechanisms that drive the spectral transitions. However, due to its faintness in recent years, it has been challenging to obtain the spectra of GR 290 with high enough quality for wind speeds to be adequately estimated.

Many LBVs are surrounded by small nebulae whose structure and chemical composition have been studied in order to investigate their past history (e.g. Martayan et al. 2016, and references therein). In this regard, Fabrika et al. (2005) claimed a marginal detection, in an $\mathrm{H} \beta$ image, of a compact elongated nebula around GR 290. However, no detailed spectrophotometric study of the nebular emission near GR 290 has been so far carried out.

In this paper we present the results of our ongoing spectral and photometric monitoring of GR 290. We report the results of observations performed in 2016 with one of the best spectral resolutions and signal-to-noise ratios ever obtained for this object. We are particularly targeting the information on the circumstellar emission around GR 290 that can be extracted from these spectra, which may give us some knowledge on the mass loss during the previous stages of the star's life.

In Sect. 2 we describe the observations; in Sect. 3 the current spectral characteristics and deduced parameters from CMFGEN fits; in Sect. 4 the spectral characteristics of the interstellar medium and OB-star regions in the neighbourhood of GR 290. In Sect. 5 we present the conclusions.

\section{Observations}

GR 290 was observed with the Optical System for Imaging and low-Intermediate-Resolution Integrated Spectroscopy (OSIRIS) 
Table 1. Spectroscopic observations 2016 July and August.

\begin{tabular}{lccrlll}
\hline \hline MJD & Filter ID & $\begin{array}{c}\lambda \text {-range } \\
(\AA)\end{array}$ & $\begin{array}{r}t_{\text {exp }} \\
(\mathrm{s})\end{array}$ & $S / N$ & $\AA$ A/pix & Res. \\
\hline 57599.16 & R2500U & $3440-4610$ & 1200 & $40-50$ & 0.62 & 2555 \\
57627.07 & $"$ & $"$ & 1200 & $20-40$ & $"$ & $"$ \\
57599.17 & R2500V & $4500-6000$ & 900 & $25-46$ & 0.80 & 2515 \\
57627.09 & $"$ & $"$ & 900 & $25-50$ & $"$ & $"$ \\
57599.18 & R2500R & $5575-7685$ & 600 & $30-37$ & 1.04 & 2475 \\
57627.10 & $"$ & $"$ & 600 & $20-27$ & $"$ & $"$ \\
\hline
\end{tabular}

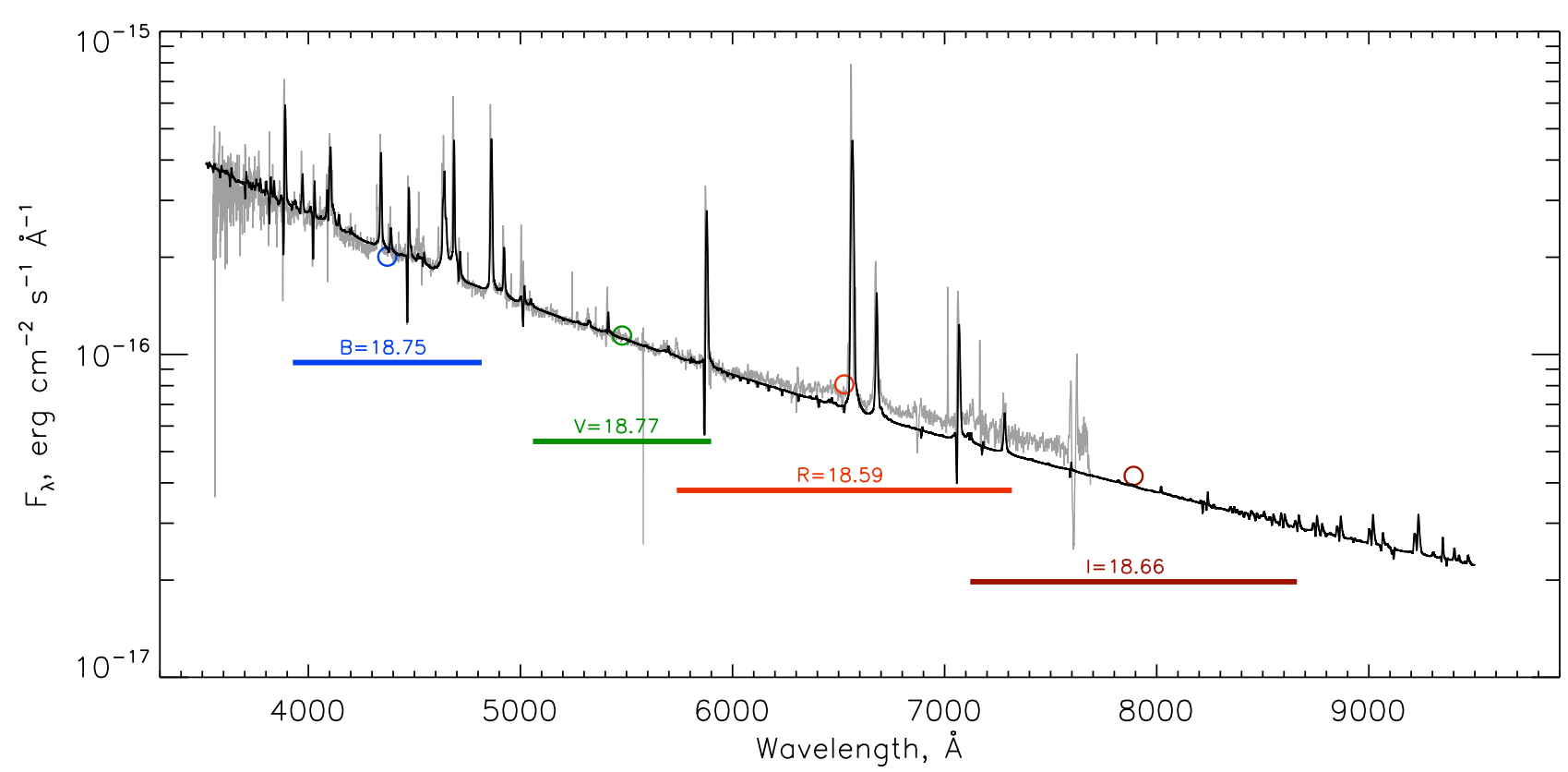

Fig. 1. Flux-calibrated spectrum of GR 290 (grey line) compared with the best-fit $\beta=2$ CMFGEN model (black line). Measured photometric magnitudes and corresponding bandwidths are indicated. The model spectrum is scaled for the distance to the M 33 and the interstellar extinction $\left(E_{(B-V)}=0.06\right)$ is applied to it. The adopted distance to M 33 is $847 \pm 61 \mathrm{kpc}$ (distance module $\left.24.64 \pm 0.15\right)$ from Galleti et al. (2004).

on the Gran Telescopio Canarias (GTC) on 2016 July 30 and August 27 (MJD 57599.1569, 57627.073, respectively) in Service Observing mode using the R2500-set of gratings and the long slit $\left(7.4^{\prime}\right)$ with a slit width of $0.6^{\prime \prime}$. These gratings cover the ultraviolet $(U)$, visual $(V)$, and red $(R)$ wavelength intervals as defined in the third column of Table 1 . This table also lists the filter identification (ID), the exposure time, the range in signal-to-noise $(\mathrm{S} / \mathrm{N})$, the spectral dispersion, and the resolution for each of the observations. Position angles were $94.75 \mathrm{deg}$ and $95.2 \mathrm{deg}$, respectively.

The spectra were pre-processed by the standard GTC/OSIRIS pipeline ${ }^{2}$ (Ederoclite \& Cepa 2010). The data were then reduced using the standard procedures from Image Reduction and Analysis Facility (IRAF) ${ }^{3}$, which included the bias and flat field corrections as well as the wavelength calibration. The root mean square error of the fit for the wavelength calibration on the comparison lamps is $0.032 \AA$ in the red and $0.015 \AA$ in visual and blue spectra. Lines lying on overlapping segments obtained from the three filters were checked for

\footnotetext{
2 http://gtc-osiris.blogspot.mx/2012/10/ the-osiris-offline-pipeline-software.html

3 IRAF is distributed by the NOAO, which is operated by AURA, under contract with NSF.
}

relative shifts. These lines are $\mathrm{He}_{\mathrm{I}} 4471 \AA$, which appears in both the $U$ and the $V$ segments, and the He I $5876 \AA$, which appears in the $V$ and $R$ segments. Both lines have PCyg profiles and we measured the emission and the absorption components separately as individual features for the purpose of verifying the wavelength calibration. We find that the $U$ and $V$ segments overlap to within $\pm 0.2 \AA$ at $\lambda 4471$ in both the July and August observations. The $V$ and $R$ segments overlap to within $\pm 0.3 \AA$ in the August observation. However, the He I $5876 \AA$ line in the $R$ segment in the July observation is shifted by about $-1 \AA$ the source of which we are unable to ascertain. Although such a significant shift is not present in other lines of this $R$ segment, we deem the radial velocities (RVs) from this segment to be less reliable than those in the others.

The flux calibration was performed using standard routines in IRAF, as well as Interactive Data Language (IDL) procedures originally written for $\mathrm{SCORPIO}^{4}$ data reduction. The resulting flux-calibrated spectra, however, still suffer from the following limitations: (1) the lack of an accurate and updated extinction curve of the site, mainly in the red and (2) the fact that the

\footnotetext{
4 SCORPIO is Spectral Camera with Optical Reducer for Photometric and Interferometric Observations (SCORPIO; Afanasiev \& Moiseev 2005) on the Russian 6-m telescope.
} 
Table 2. Photometry obtained on 2016 July 31 using the JohnsonCousins filters.

\begin{tabular}{lllrl}
\hline \hline Band & Magnitude & \pm & $S / N$ & UT \\
\hline$V$ & 18.77 & 0.04 & 71 & $01: 44: 14$ \\
$R$ & 18.59 & 0.04 & 72 & $01: 53: 10$ \\
$I$ & 18.66 & 0.05 & 46 & $02: 00: 57$ \\
$B$ & 18.75 & 0.03 & 99 & $02: 10: 43$ \\
\hline
\end{tabular}


Fig. 2. Left: line profiles of He II plotted on a velocity scale corrected for the adopted $-179 \mathrm{~km} \mathrm{~s}^{-1}$ systemic velocity. The continuous line shows the strong $\lambda 4686$, with its extended electron scattering wings, and $\lambda 5411$ with its strong PCyg absorption. The dashed line is $\lambda 4541$, which is contaminated on the blue wing with N III. Right: He I lines profiles of $\lambda 4471$ (continuous, black), $\lambda 5876$ (dash, black), $\lambda 6678$ (dot, red), and $\lambda 7065$ (continuous, red). The vertical lines indicate the approximate locations where the line meets the continuum level.

standard star that was used is more than ten magnitudes brighter than GR 290 so that the instrument was de-focused in order to avoid saturation. The absolute fluxes (shown in Fig. 1) are thus to be considered only approximate.

Photometric observations in the Johnson-Cousins filters were obtained using the 1.52-m Cassini Telescope run by INAF-Osservatorio Astronomico di Bologna in Loiano on 2016 July 31 , providing a photometric reference for the GTC July 30 observation. Table 2 lists in Col. 1 the wavelength band, in Col. 2 the magnitude value, in Cols. 3 and 4 the measurement uncertainty and the $\mathrm{S} / \mathrm{N}$, respectively, and in Col. 5 the Universal Time (UT) of the observations. With $m_{\mathrm{V}}=18.8 \mathrm{mag}$, GR 290 is currently at its faintest since the beginning of the twentieth century (see Polcaro et al. 2016 for the photometric history).

\section{Spectrum of GR 290}

The flux calibrated spectrum of GR 290 is characterized by a blueward rising continuum and strong emission lines, as illustrated in Fig. 1. In addition to $\mathrm{H} \alpha$ and $\mathrm{H} \beta$, the spectrum is dominated by lines from He I, He II, and N III. The P Cyg absorption components of the $\mathrm{He}$ I triplet lines are enhanced with respect to the singlets, due to the overpopulation with respect to local thermodynamical equilibrium (LTE) of the low-lying triplet levels in the low density stellar wind. The He II Paschen-like line at $4686 \AA(3-4)$ is observed in emission, as in earlier spectra, while the multiplet two Brackett-like lines at $5411 \AA$ (47) and $4541 \AA$ (4-9) have P Cyg profiles and are now stronger than in earlier spectra. The He II line from this same series at $4199 \AA$ (4-11) (blended with N III) also displays a PCyg profile. The profiles of some of the He II lines are illustrated in Fig. 2.
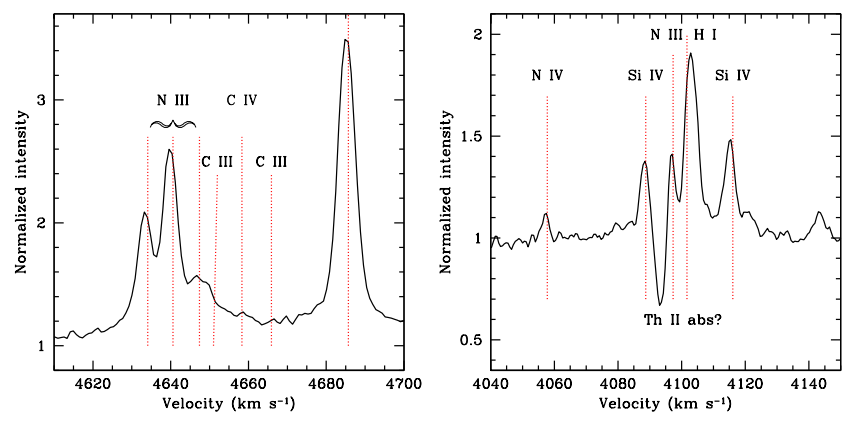

Fig. 3. Line profiles on a wavelength scale corrected for the adopted $-179 \mathrm{~km} \mathrm{~s}^{-1}$ systemic velocity of M33. Left: N III 4634-41 and He II 4686 emission lines. We note the possible contribution of CIV. Right: N IV 4058 and the $4100 \AA$ blend that includes Si IV, N III, and H I lines. Vertical lines indicate the laboratory wavelength of the transitions.

The strongest N III lines are those at $4634-41 \AA$, which are partially resolved (Fig. 3, left). There is a prominent absorption blueward of $\mathrm{N}$ III $4097 \AA$ suggesting that this line also has a P Cyg profile. The $\mathrm{N}_{\text {III }} 4196,4200$, and $4379 \AA$ are also now present, consistent with a higher degree of ionization in the wind suggested by the increased strength of He II 5411. Most importantly, we note the presence for the first time of N IV $4058 \AA$ in Fig. 3 (right). It is clearly present in both the July and the August spectra and has an equivalent width of $0.5 \AA$ on the combined spectrum. The N IV lines at 7109 and $7122 \AA$ appear to also be weakly present.

The primary indicator of ionization classification given by Smith et al. (1996) is the ratio of equivalent widths (EW) of He II $5411 / \mathrm{He}$ I $5876 \AA$. We find that this ratio is $\leq 0.1$, implying a degree of ionization corresponding to WN8. The earlier criteria of van der Hucht et al. (1981), which are based on the Smith (1968) system, rely on the ratios of $\mathrm{N}$ III/N IV/N v 4634-4641, 4057, and 4604-4620 ̊, respectively. The fact that EW(N III) $\gg \mathrm{EW}(\mathrm{N}$ IV) is consistent with WN8 or cooler (see Table 3).

\subsection{Radial velocities}

The radial velocity (RV) and full-width at half maximum intensity (FWHM) was obtained through a Gaussian fit to individual line profiles or, in the case of overlapping lines and PCyg profiles, through simultaneous multiple Gaussian fits. Table 3 contains the results of our measurements. Columns 1 and 2 list the primary atomic identification and corresponding laboratory wavelength; Cols. 3 and 5 list the heliocentric RV of the line centroid for the July and the August observations, respectively. The corresponding heliocentric corrections are $+27 \mathrm{~km} \mathrm{~s}^{-1}$ for July and $+24 \mathrm{~km} \mathrm{~s}^{-1}$ for August. Columns 4 and 6 list the corresponding values of the FWHM.

In order to obtain the velocity of GR 290 with respect to M33, we averaged the RVs of the lines in Table 3 marked with an asterisk. The selection of these lines is based on the following criteria: (1) relatively unblended line; (2) relatively good S/N; (3) little or no ambiguity in the identification. We find the averages to be $\operatorname{RV}(J u l y)=-171 \pm 22$ (s.d.) $\mathrm{km} \mathrm{s}^{-1}$ and $\mathrm{RV}$ (August) $=-155 \pm 24$ (s.d.) $\mathrm{km} \mathrm{s}^{-1}$. Averaging the RVs of July and August yields RV(GR 290) $=-163 \pm 32 \mathrm{~km} \mathrm{~s}^{-1}$. The three averages are consistent, within the uncertainties, with each other and with the heliocentric velocity $-179 \pm 3 \mathrm{~km} \mathrm{~s}^{-1}$ of M 335. We thus adopt this latter value for the systemic velocity

\footnotetext{
5 NASA/IPAC/JPL extragalactic database.
} 
Table 3. Emission line radial velocity, FWHM, and equivalent width measurements.

\begin{tabular}{|c|c|c|c|c|c|c|c|c|c|}
\hline & \multirow[b]{2}{*}{ ID } & \multicolumn{2}{|c|}{ July } & \multicolumn{2}{|c|}{ August } & \multicolumn{4}{|c|}{ Average } \\
\hline & & RV & FWHM & RV & FWHM & $\mathrm{RV}_{\mathrm{em}}$ & $-\mathrm{EW}$ & $V_{\mathrm{abs}}$ & $V_{\text {edge }}$ \\
\hline $\mathrm{He}_{\mathrm{I}}$ & $3704.00 ?$ & -15 & 332. & -56 & 307. & 135 & 1.1 & -210 & -250 \\
\hline $\mathrm{HI}$ & 3750.20 & -129 & 328. & $\ldots$ & $\ldots$ & 16 & 0.6 & -172 & -283 : \\
\hline $\mathrm{HI}_{\mathrm{I}}$ & 3770.60 & -102 . & 358. & -85 & 374. & 75 & 1.8 & -258 & $-400:$ \\
\hline $\mathrm{HI}$ & 3797.90 & -105 & 308. & $\ldots$ & $\ldots$ & -10 & 0.4 & -221 & -310 \\
\hline $\mathrm{He}_{\mathrm{I}}$ & $3819.60 *$ & -178 & 361. & -136 & 228. & 17 & 1.2 & -252 & -530 \\
\hline H I & $3835.40 *$ & -147 & 320. & -104 & 461. & 32 & 1.2 & -201 & -370 : \\
\hline $\mathrm{He}$ I & 3888.65 & -149 & 457 & -183 & 485: & 20 & 10.4 & -350 & -660 \\
\hline $\mathrm{HI}$ & $3970.10 *$ & -124 & 325. & -149 & 310. & 18 & 2.2 & -193 & \\
\hline $\mathrm{He}$ I & $4026.20 *$ & -149 . & 342. & -152 & 342. & 10 & 2.4 & -248 & -550 \\
\hline N IV & 4057.76 & -223 & 273 & -164 & 148. & -10 & 0.5 & $\ldots$ & $\ldots$ \\
\hline Si IV & $4088.70 *$ & -182 & 213. & -173 & 198. & 2 & 1.7 & $\ldots$ & $\ldots$ \\
\hline N III & 4097.30 & -200 & 154. & -190 & $\ldots$ & -4 & 2.0: & -230 & -438 \\
\hline $\mathrm{HI}_{\mathrm{I}}$ & 4101.74 & -67 & 373. & -51 & 285. & 121: & 5.2 & $\ldots$ & $\ldots$ \\
\hline Si IV & $4116.10 *$ & -228 & 364. & -211 & 233. & -32 & 2.8 & $\ldots$ & $\ldots$ \\
\hline N III & 4195.76 & -234 & 141. & -266 & 164. & $-66:$ & 0.2 & $\ldots$ & $\ldots$ \\
\hline N III & 4200.10 & -162. & 206. & -165 & 142. & 13 & 0.6 & $-159:$ & $-260:$ \\
\hline $\mathrm{HI}$ & $4340.50 *$ & -149 & 380. & -119 & 366. & 42 & 7.9 & $\ldots$ & $\ldots$ \\
\hline N III & $4379.20 *$ & -186. & 260. & -164 & 226. & -7 & 1.0 & $\ldots$ & $\ldots$ \\
\hline $\mathrm{He}$ I & $4387.90 *$ & -198. & 301. & -135 & 246. & 13 & 1.6 & $\ldots$ & $\ldots$ \\
\hline $\mathrm{He}$ I & $4471.50 *$ & -149 & 389. & -127 & 362. & 28 & 4.9 & -275 & -630 \\
\hline N III & $4534.60 *$ & -194. & 178. & -149 & 165. & -22 & $0.4:$ & $-233:$ & -280 : \\
\hline He II & 4541.60 & -145 & 145. & $\ldots$ & $\ldots$ & 29: & 2.0: & -182 & -300 : \\
\hline $\mathrm{N}_{\text {III }}$ & 4634.14 & -183 & 256 & -188 & 252 & -9 & 5.5 & $\ldots$ & $\ldots$ \\
\hline N III & 4640.64 & -197. & 252 & -195 & 258. & -16 & 9.4 & $\ldots$ & $\ldots$ \\
\hline He II & $4685.6^{*}$ & -176 & 333. & -174 & 326. & 7 & 17.4 & $\ldots$ & $\ldots$ \\
\hline $\mathrm{He}$ I & $4713.1 *$ & -146 & 197. & -130 & 407. & 28 & 1.6: & -291 & -500 : \\
\hline $\mathrm{HI}$ & $4861.3 *$ & -171 & 395. & -167 & 401. & 11 & 18.2 & $\ldots$ & $\ldots$ \\
\hline $\mathrm{He}$ I & $4921.9 *$ & -185 & 335. & -174 & 371. & 4 & 4.3 & $\ldots$ & $\ldots$ \\
\hline $\mathrm{He} \mathrm{I}$ & $5015.7 *$ & -162 & 275. & -149 & 287. & 15 & 1.7 & $-200:$ & $\ldots$ \\
\hline $\mathrm{He}_{\mathrm{I}}$ & $5047.7 *$ & -174 & 184. & -143 & 237. & 41 & 0.6 & $\ldots$ & $\ldots$ \\
\hline He II & 5411.52 & -150 & 260 & -169 & 294. & 15 & 1.9 & -217 & -433 \\
\hline $\mathrm{He} \mathrm{I}$ & $5875.66^{*}$ & -175 & 444. & -171 & 464. & 5 & 24.5 & -304 & -655 \\
\hline $\mathrm{HI}$ & 6562.80 & -184 & 301. & -176 & 269. & -1 & $\ldots$ & $\ldots$ & $\ldots$ \\
\hline $\mathrm{HI}$ & $6562.80 *$ & -181 & 466. & -172 & 461. & 8 & 68.0 & $\ldots$ & $\ldots$ \\
\hline $\mathrm{He} \mathrm{I}$ & $6678.15^{*}$ & -176 & 417. & -185 & 395. & -6 & 18.0 & $\ldots$ & $\ldots$ \\
\hline $\mathrm{He}$ I & $7065.20 *$ & -152 . & 467. & -157 & 450. & 13 & 18.1 & -306 & -560 \\
\hline N IV & 7109.35 & -169 & 181. & $\ldots$ & $\ldots$ & 8 & $0.2:$ & $\ldots$ & $\ldots$ \\
\hline N IV & 7122.98 & -160 & 231. & $\ldots$ & $\ldots$ & $\ldots$ & 0.3 & $\ldots$ & $\ldots$ \\
\hline
\end{tabular}

Notes. Column 1: primary atomic identification and corresponding laboratory wavelength in $\AA$; Cols. 2 and 4: RVs for the July and the August observations respectively, after applying the heliocentric corrections of $+27 \mathrm{~km} \mathrm{~s}^{-1}$ for July and $+24 \mathrm{~km} \mathrm{~s}^{-1}$ for of August; Cols. 3 and 5: corresponding full-widths at half maximum intensity. Columns 6-9: measurements performed on the combined July+August spectrum after correcting for the adopted $-179 \mathrm{~km} \mathrm{~s}^{-1}$ systemic velocity of M33. Column 6: RV of the emission lines; Col. 7: corresponding equivalent width; Col. 8: centroid of the P Cyg absorption component when present; and Col. 9: velocity of the P Cyg absorption edge (i.e. the location where the absorption reaches the continuum). RVs and FWHM's are in units of $\mathrm{km} \mathrm{s}^{-1}$; EWs are in units of $\AA$, and the negative sign in the column heading indicates emission. Asterisk indicates the line was used to determine the RV of the system.

of GR 290 in the remainder of this paper. The standard deviations are of similar value to our estimated measurement uncertainty of $\pm 30 \mathrm{~km} \mathrm{~s}^{-1}$.

Since we do not detect variability in our two spectra that is larger than the uncertainty discussed above, we combined the July and August spectra, shifted the average by $+179 \mathrm{~km} \mathrm{~s}^{-1}$, and remeasured the lines. We list in Table 3 the RV of the emission in Col. 7, the equivalent width in Col. 8, the RV of the absorption component in the case of P Cyg profiles in Col. 9, and the intersection of the P Cyg absorption with the continuum level in Col. 10. The measurements for P Cyg profiles were performed with two-Gaussian fits ${ }^{6}$. We note that the contribution of nebular emission affects the FWHM. For example, in the case of $\mathrm{H} \alpha$, we measure $F W H M=6.6 \AA$ if the line is fit with a single Gaussian while $F W H M=10.2 \AA$ if the line is treated as a blend of a broad

\footnotetext{
6 It is important to note that two-Gaussian fits to PCyg profiles lead to a significant difference in the centroid and FWHM compared to a procedure in which the emission and absorption components are each measured independently. This is because when deblending, the combined profile is fitted with an absorption and a neighbouring, partially overlapping emission. This results in an emission component having a bluer RV and a broader FWHM than if it were fit individually.
} 


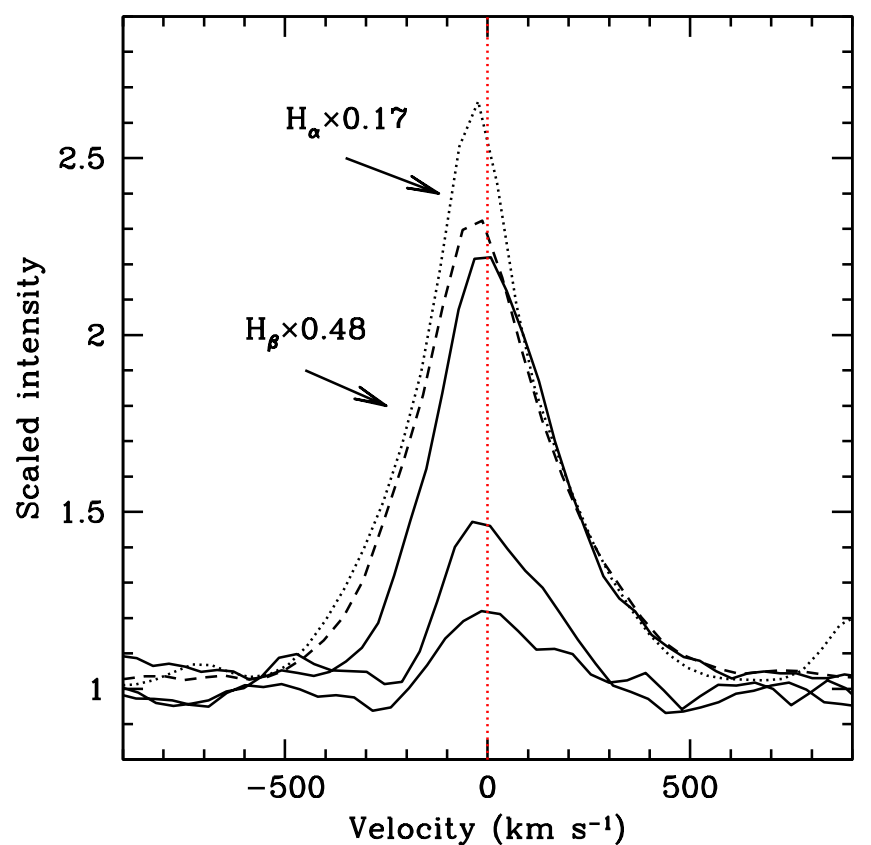

Fig. 4. Line profiles of the Balmer-H sequence plotted on a velocity scale corrected for the adopted $-179 \mathrm{~km} \mathrm{~s}^{-1}$ systemic velocity. The lines of $\mathrm{H} \alpha$ and $\mathrm{H} \beta$ are scaled by factors of 0.17 and 0.48 , respectively, so as to align the red wings with that of $\mathrm{H} \gamma$. The continuous lines show $\mathrm{H} \gamma$, $\mathrm{H} \epsilon$, and H I $3835 \AA$. The progressive red-shift at shorter wavelengths appears to be a consequence of the increasing strength of the blue-shifted absorption which, in the helium lines, takes on the form of PCyg absorption. This effect is explained by the fact that the optical depth of the wind is highest for $\mathrm{H} \alpha$ and $\mathrm{H} \beta$ and decreases for lines that have smaller transition probabilities.

stellar line and a narrow superposed nebular emission. Despite this large difference in FWHM, the RV in both cases is nearly the same.

The average $V_{\text {edge }}=-400 \pm 144 \mathrm{~km} \mathrm{~s}^{-1}$ is similar to the value of $v_{\infty}$ listed in Polcaro et al. (2016) for recent spectra. However, the He I lines have $V_{\text {edge }} \simeq 620 \mathrm{~km} \mathrm{~s}^{-1}$, implying wind speeds that are faster than ever before observed.

The Balmer-H lines also show the signs of a relatively fast outflow, as can be seen from the growing asymmetry in the blue wing of the higher excitation lines of this series. This is illustrated in Fig. 4 where $\mathrm{H} \alpha$ and $\mathrm{H} \beta$ were scaled in intensity so as to align the red wings with that of $\mathrm{H} \gamma$. The other lines plotted are $\mathrm{H} \epsilon$ and $\mathrm{H}_{\mathrm{I}} 3835 \AA$. This asymmetry results from the same process that leads to P Cyg absorptions in He I but because of the large optical depth of the $\mathrm{H}$-lines a fully developed absorption component is not visible.

\subsection{Stellar wind models}

We used the CMFGEN atmospheric modelling code (Hillier \& Miller 1998) in order to estimate the current stellar parameters of GR 290. This code solves the radiative transfer equation for objects with spherically symmetric extended outflows using either the Sobolev approximation or the full comoving-frame solution of the radiative transfer equation. CMFGEN incorporates line blanketing, the effect of Auger ionization and clumping, which is parametrized by the volume filling factor $f=\bar{\rho} / \rho(r)$, where $\bar{\rho}$ is the homogeneous (unclumped) wind density and $\rho$ is the density inside clumps assumed to be optically thin to radiation, while the interclump medium is void (Hillier \& Miller 1999). Every
Table 4. Chemical abundances in the CMFGEN model.

\begin{tabular}{lllll}
\hline \hline Species & Rel. Num. Frac. & Mass Frac & $\mathrm{NH}$ & $Z / Z_{\odot}$ \\
\hline $\mathrm{H}$ & 2.2 & 0.35 & 12 & 0.5 \\
$\mathrm{He}$ & 1.0 & 0.64 & 11.66 & 2.3 \\
$\mathrm{C}$ & $1 \times 10^{-4}$ & $2 \times 10^{-4}$ & 7.66 & 0.06 \\
$\mathrm{~N}$ & $1.5 \times 10^{-3}$ & $3.5 \times 10^{-3}$ & 8.83 & 3.0 \\
$\mathrm{O}$ & $1 \times 10^{-4}$ & $2.5 \times 10^{-4}$ & 7.66 & 0.03 \\
& & & & \\
$\mathrm{Ne}$ & $2.4 \times 10^{-4}$ & $7.8 \times 10^{-4}$ & 8.04 & 0.45 \\
$\mathrm{Mg}$ & $5.0 \times 10^{-5}$ & $2.0 \times 10^{-4}$ & 7.37 & 0.3 \\
$\mathrm{Al}$ & $8.4 \times 10^{-6}$ & $3.7 \times 10^{-5}$ & 6.58 & 0.65 \\
$\mathrm{Si}$ & $3.0 \times 10^{-5}$ & $1.4 \times 10^{-4}$ & 7.14 & 0.2 \\
$\mathrm{~S}$ & $2.3 \times 10^{-5}$ & $1.2 \times 10^{-4}$ & 7.02 & 0.32 \\
$\mathrm{Ar}$ & $6.5 \times 10^{-6}$ & $4.2 \times 10^{-5}$ & 6.47 & 0.4 \\
$\mathrm{Ca}$ & $4.2 \times 10^{-6}$ & $2.7 \times 10^{-5}$ & 6.28 & 0.4 \\
$\mathrm{Fe}$ & $4.9 \times 10^{-5}$ & $4.4 \times 10^{-4}$ & 7.4 & 0.32 \\
\hline
\end{tabular}

model is defined by a hydrostatic stellar radius $R_{*}$, luminosity $L_{*}$, mass-loss rate $\dot{M}$, filling factor $f$, wind terminal velocity $v_{\infty}$, stellar mass $M$, and by the abundances $Z_{i}$ of the chemical elements that are included. LBVs may have inflated radii and pseudophotospheres (Gräfener et al. 2012), which may cause large uncertainties in the $R_{*}$ value derived from CMFGEN. Thus, hydrodynamical models need to be used to compute the hydrostatic radius and when discussing the evolutionary status of these objects, which goes beyond the scope of our current investigation.

The CMFGEN models were computed in an iterative manner, starting from an initial model and then adjusting parameters until a reasonable match between the model and observed spectra was attained. The temperature $T_{\mathrm{eff}}{ }^{7}$ was first adjusted by using the ratios of $\mathrm{He} I / / \mathrm{He}$ I and $\mathrm{N}$ II $/ \mathrm{N}$ III/ $/ \mathrm{N}$ IV. Then the luminosity was adjusted by comparing with the contemporary $V$-band and $B-V$ photometry (see Table 2). The value of $\dot{M}$ was obtained from the line intensities, and the $v_{\infty}$ from the P Cyg profile shapes. The filling factor $f=0.15$ was determined by fitting emission line profiles.

The abundances of chemical elements used in the models are listed in Table 4 . The relative number fraction is given in Col. 2, the corresponding mass fraction in Col. 3, the adopted solar abundance in Col. 4 and the model abundance relative to the solar abundance in Col. 5. The abundances of $\mathrm{H}, \mathrm{He}, \mathrm{C}, \mathrm{N}$, and $\mathrm{O}$ were estimated from the model, while for the abundances of Ar, S, and Ne we used those of M 33 from Magrini et al. (2010). For the abundances of other elements $(\mathrm{Mg}, \mathrm{Al}, \mathrm{Si}, \mathrm{Ca}$, and $\mathrm{Fe}$ ) we assumed values of half solar as the metallicity of the outer regions of M 33 is low.

Beta velocity law $v(r)=v_{\infty}\left(1-\frac{R_{*}}{r}\right)^{\beta}$ approximation is one of the basic simplifications typically adopted while constructing atmospheric models of hot stars. Radiation-driven wind theory (Puls et al. 1996; Lamers \& Cassinelli 1999) predicts the values of $\beta=0.5-1$, while more hydrodynamically consistent numerical models favour $\beta=2.4$ for inner parts of the wind, and $\beta=0.9$ - for the outer ones (Sander et al. 2017). In our previous paper by Polcaro et al. (2016) we built the models assuming $\beta=1$, as our main goal was to compare the spectra acquired with different spectral resolutions and covering

\footnotetext{
7 Here $T_{*}$ and $T_{\text {eff }}$ are effective temperatures at the bottom of the wind
} and at radius $R_{2 / 3}$ where Rosseland optical depth is $2 / 3$. 



Fig. 5. Effect of increasing $\beta$-parameter in the velocity law on spectral line profiles. From left to right: modelled profiles of $\mathrm{H} \beta, \mathrm{H} \alpha$, and $\mathrm{He}$ II 5411 . For comparison, the actual profiles observed in the spectrum of GR 290 are shown by a black dashed line.

Table 5. CMFGEN model fit results.

\begin{tabular}{llll}
\hline \hline Quantity & Model 1 & Model 2 & Note \\
\hline$L_{*} / 10^{5} \times L_{\odot}$ & $(3.7 \pm 0.2)$ & $(3.1 \pm 0.2)$ & \\
$\log \left(L_{*} / L_{\odot}\right)$ & $5.57 \pm 0.03$ & $5.49 \pm 0.03$ & \\
$\dot{M} / 10^{-5} \times M_{\odot} \mathrm{yr}^{-1}$ & $(1.5 \pm 0.2)$ & $(1.4 \pm 0.2)$ & \\
$R_{*} / R_{\odot}$ & $15-16.5$ & $13.5-15.2$ & $(\mathrm{a})$ \\
$T_{*} / \mathrm{K}$ & $36000 \pm 500$ & $36000 \pm 500$ & $(\mathrm{a})$ \\
$R_{2 / 3} / R_{\odot}$ & $21-24.2$ & $22.6-26.6$ & $(\mathrm{~b})$ \\
$T_{\mathrm{eff}} / \mathrm{K}$ & $30000 \pm 700$ & $27500 \pm 700$ & $(\mathrm{~b})$ \\
$v_{\infty} / \mathrm{km} \mathrm{s}^{-1}$ & $620 \pm 50$ & $620 \pm 50$ & \\
$\beta$ & 2.0 & 3.0 & \\
\hline
\end{tabular}

Notes. (a) The radius and temperature at the bottom of the wind where $\tau=18.7$; (b) the temperature and radius at $\tau=2 / 3$.

different spectral ranges in order to track the evolution of temperature, luminosity, and mass-loss rate over time. In the present article we are studying the spectrum with much better resolution covering the whole visible range, and immediately after starting the modelling process we found that the profiles of hydrogen emission lines are not consistent with $\beta=1$. Therefore we had to build the models for several $\beta$ values. Figure 5 illustrates the changes of the lines' profiles with increasing $\beta$ (Table 5).

The models with $\beta=2$ and $\beta=3$ are closest to reproducing the observed spectrum. Both models provide a satisfactory fit to the overall spectral energy distribution and results of multicolour photometry as seen in Fig. 1, where the apparent discrepancy to the right of $\sim 6300 \AA$ is caused by the uncertainties in the flux calibration and the adopted reddening correction, as well as the strong emission line here. The detailed comparison of the observed spectrum with the best model with $\beta=2$ is shown in Fig. 6. Figure 7 clearly demonstrates that some lines are better described with $\beta=2$, while others - with $\beta=3$.

The models coincide relatively well with the broad portion of the $\mathrm{H} \alpha, \mathrm{H} \beta$ profiles, lying below the observed line maximum. This is consistent with the fact that GR 290 lies within a nebular region from which significant $\mathrm{H}$-Balmer emission arises and which is responsible for the sharp superposed emission in these lines (see Sect. 4 below). The emission component of the He I PCyg lines is well-reproduced by the models, but not so the absorption component, which tends to be weaker than predicted.
None of the models reproduces the profile shape of the highexcitation He II $5411 \AA$ line, and this constitutes the largest discrepancy between the models and the observation. The model emission-line profile is a factor of approximately two narrower and the absorption component is significantly less extended than the observations. Because of its high excitation, this line generally originates close to the hydrostatic photosphere where the wind is still accelerating. This discrepancy suggests that the emission-line-forming region in GR 290 may be more complex than assumed in the model. That is, it may not be spherically symmetrical, or portions of the wind emission may be dominated by shock emission, factors which are not incorporated in the current CMFGEN model.

\section{The nebula surrounding GR 290}

Circumstellar gaseous nebulae are formed around massive stars during BSG (Lamers et al. 2001) and LBV stages (Weis 2012). The sizes of these nebulae significantly increase under the effect of fast stellar wind when the central stars transit to the WR stage. Nebulae can tell us the history of their stars' formation, ages, and masses of ejected material. The presence of forbidden lines in the spectra of GR 290 indicates that it has a nebula, but the large distance prevents its detection on direct images. Below we investigate the structure of GR 290's nebula using its twodimensional spectrum.

The two-dimensional spectral image obtained with GTC contains information on the interstellar medium in the neighbourhood of GR 290. The long OSIRIS slit was oriented in the east-west direction and in addition to GR 290, crosses the nearby OB88 and OB89 associations (Humphreys \& Sandage 1980; Ivanov et al. 1993; Massey et al. 1995). OB89 and OB88 lie west of GR 290.

Figure 8 illustrates the tracing in the spatial dimension along the $\mathrm{H} \alpha$ emission line. GR290 is at the origin of coordinates and appears sharp and narrow compared to the other features in this tracing. From an analogous tracing at $[\mathrm{N} \mathrm{II}] 6548 \AA$, the spatial resolution is estimated to be approximately four pixels, which is approximately $4 \mathrm{pc}$ assuming a M 33 distance of $847 \mathrm{kpc}$. This is consistent with the limit imposed by the seeing of the observations: $\sim 1.2^{\prime \prime}$ in July and $\sim 1.6^{\prime \prime}$ in August.

A Gaussian fit to the sharp stellar emission peak indicates that there is considerable $\mathrm{H} \alpha$ emission excess outside the wings 

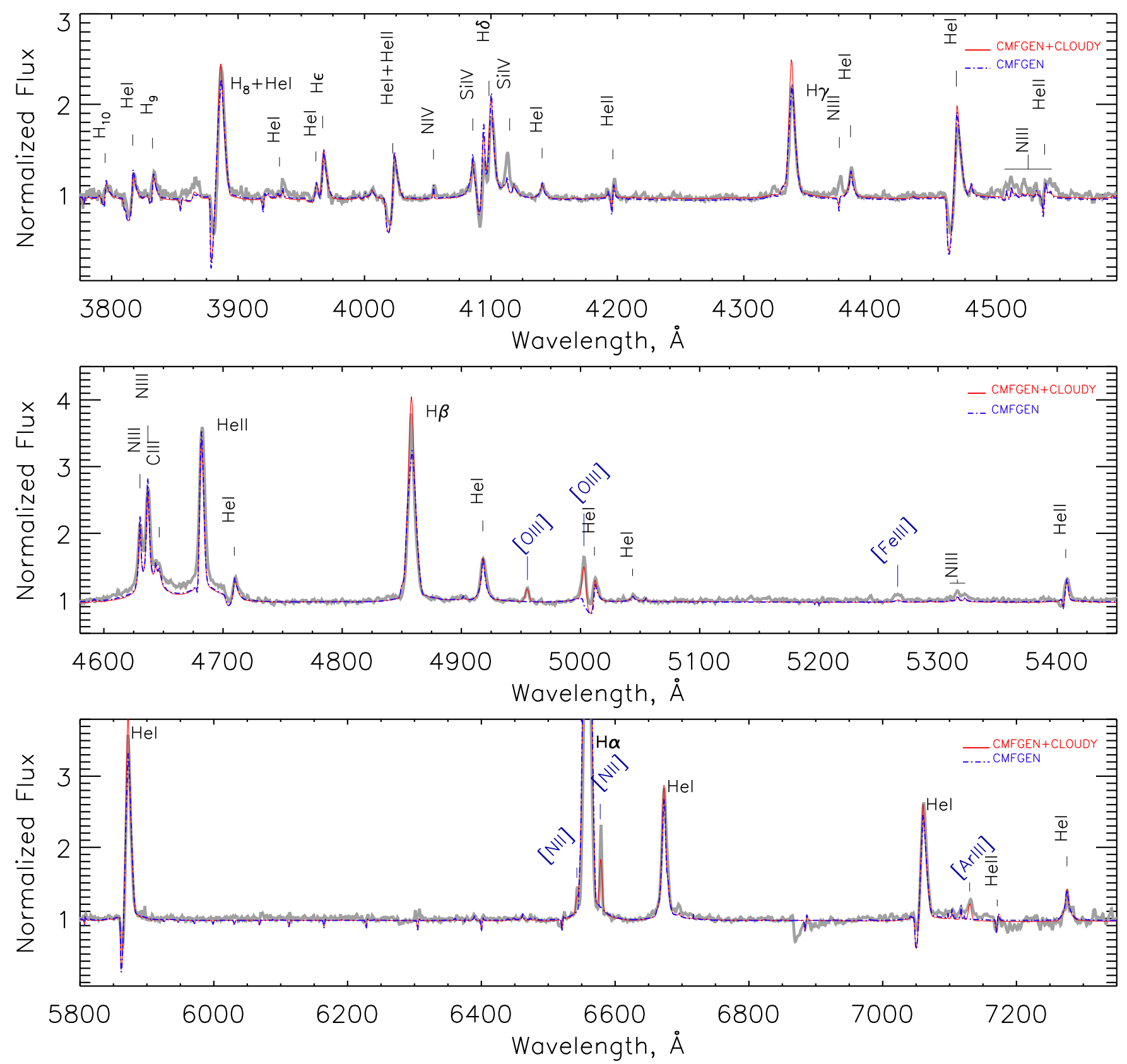

Fig. 6. Normalized optical spectrum of GR 290 (grey line) compared with the best-fit CMFGEN model with $\beta=2$ (blue dash-dotted line). Red solid line shows the sum of CMFGEN (stellar atmosphere) and CLOUDY (surrounding nebula) models. The CLOUDY model has an increased value of oxygen abundance (in comparison with the CMFGEN model), which we discuss in the text.

of this fit, which can be associated with interstellar (ISM) emission. Specifically, the $\mathrm{H} \alpha$ emission appears to extend for nine pixels ${ }^{8}$ on the eastern side of the star but only for approximately four pixels on the western side.

The RVs of the more prominent ISM lines in the twodimensional image are listed in Table 6 . The measurements were performed at three locations along the spatial resolution axis. Position 1: centred on GR 290; Position 2: on the background close to GR 290, which provides information on the diffuse component of M 33 in the neighbourhood of GR 290; and Position 3: centred on the OB89 cluster. Column 1 lists the principal

\footnotetext{
8 The "plate scale" is approximately $0.29 \mathrm{arcsec} / \mathrm{pix}$ or, assuming $D_{\mathrm{M}} 33=847 \mathrm{kpc}, 1.2 \mathrm{pc} \mathrm{pixel}^{-1}$.
}

emission line identification with its corresponding laboratory wavelength $(\AA)$; and Cols. 2-4 list the heliocentric RVs of the lines at Positions 1,2 , and 3 , respectively. The averages $( \pm$ s.d.) of the lines at the three positions are, respectively $-195 \pm 14$, $-196 \pm 36$, and $-208 \pm 19 \mathrm{~km} \mathrm{~s}^{-1}$, all consistent within the uncertainties with each other and with the systemic speed of M33. The [N II]6548 is quite extended and weak in the M33background surrounding GR 290 rendering its RV more uncertain than the rest. If we eliminate this line, the average RV for this region is $-211 \pm 20 \mathrm{~km} \mathrm{~s}^{-1}$.

$\mathrm{H} \alpha$ is present at all three positions. The [S II] 6717, 6731 lines are absent at Position 1 and the [O III] 4959, $5007 \AA$ lines are absent at Positions 2 and 3 . He I is not detected at Position 2. The spectrum at Position 1 also displays lines of [Fe III] 5270 and [Ar III] 7136. 

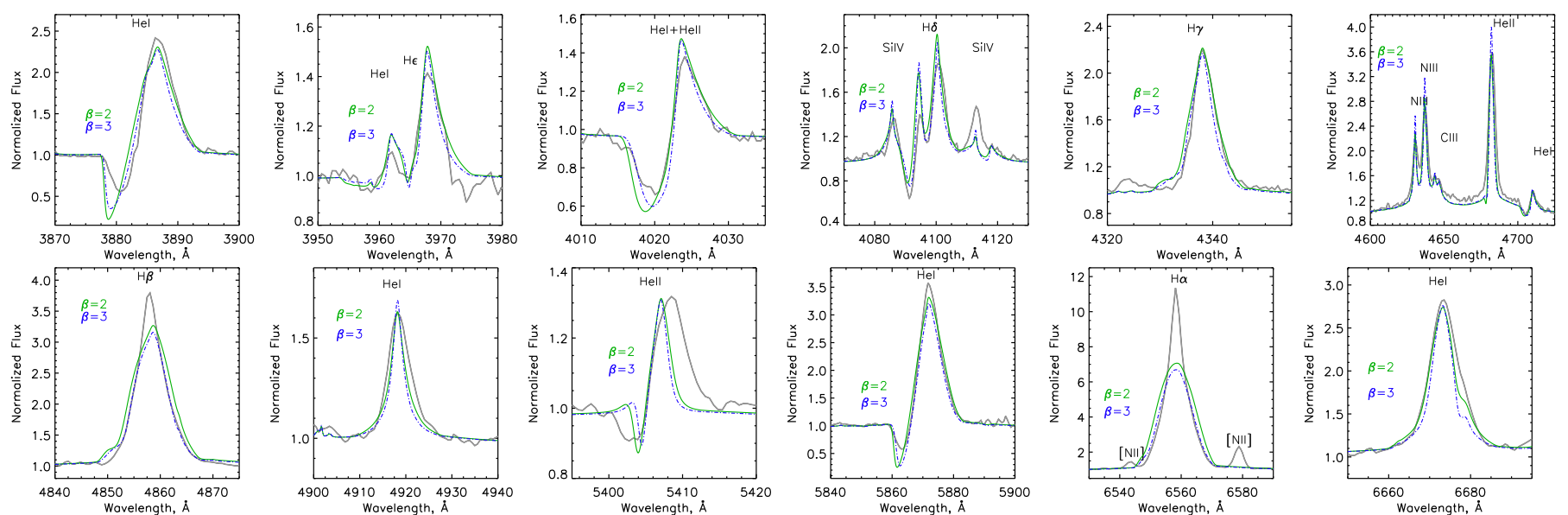

Fig. 7. Comparison of the profiles of selected lines with the best model spectra.

We used the CLOUDY (version 17.00) photoionization code by Ferland et al. $(1998,2017)$ to reveal the properties of the nebula surrounding the GR 290 star. More than a thousand models were computed, varying chemical composition, density, and outer radius of the nebula. The parameters of our best CMFGEN model were used to set the luminosity, $T_{\text {eff }}$, and spectral energy distribution of the central ionizing source. We adopted a closed spherical geometry of the nebula with the same filling factor as for the star atmosphere.

Two scenarios for the nebular chemical composition were considered. It could be formed from the surrounding material or from the LBV ejecta. For the first scenario we assumed that the chemical composition of the compact ISM region is as usually found for $\mathrm{H}$ II regions, scaled to the metallicity of M33 at the galactocentric distance of GR 290 $\left(12+\log (\mathrm{O} / \mathrm{H}) \simeq 8.15\right.$, or $\left.Z \simeq 0.3 Z_{\odot}\right)$, given the metallicity gradient derived by Rosolowsky \& Simon (2008) and Bresolin (2011). We also computed the models for 0.1, 0.2, 0.4, and $0.5 Z_{\odot}$. In the second scenario we assumed that the compact nebula was formed primarily from LBV ejecta, and we adopted the chemical composition deduced from the CMFGEN model (Table 4).

For each set of abundances, the hydrogen density was varied in the range $\log \left(n_{\mathrm{H}}\right)=1.0-3.6$ and the outer radius was varied in the $R=0.5-6 \mathrm{pc}$. We also considered larger densities and radii, but the corresponding models failed completely to fit the observed spectrum.

The aim of the modelling was to reproduce the observed nebular emission lines that are clearly seen in observed spectrum and not predicted by the CMFGEN model ([N II] 6548, 6584; [O III] 4959, 5007; [Fe III] 5270). These lines are assumed to arise in the the circumstellar medium that is photoionized by GR 290. An additional constraint on the model is the absence of the [S II] 6717, $6731 \AA$ A lines. The observed and modelled fluxes of these lines were compared and a chi-square value for each model was computed. The sum of CMFGEN model spectrum and CLOUDY model spectrum is presented in Fig. 6.

We find that models for a nebula that has the same chemical composition as the LBV stellar atmosphere yield chi-square values that are several times smaller than the models in which the alternative set of abundances are used. This result favours the hypothesis that the nebula around GR 290 has been formed primarily from material ejected from the star. Another finding is that only relatively small outer radii yield spectra similar to that observed: for $R>4 \mathrm{pc}$ the nebular emission lines become much brighter than observed, at any density and chemical abundance. This effect is clearly seen in Fig. 9, where the chi-square distribution for different densities and radii is shown and where the abundances are held constant. Although the values of the chisquared distribution may vary for different sets of abundances, the general trend is the same.

The minimum chi-square from all the computed models is obtained for an outer radius $R=0.8 \mathrm{pc}$ and a hydrogen density $n_{\mathrm{H}}=160 \mathrm{~cm}^{-3}$. Unfortunately, the spatial resolution of our observations is limited by the seeing ( 1"), which at the distance of M 33 implies that we cannot resolve structures smaller than $4 \mathrm{pc}$ in extension.

All the computed models reproduce approximately the observations if the radius of this nebula does not exceed $4 \mathrm{pc}$, with a preferred diameter being $2 \mathrm{pc}$. A larger nebula would produce brighter emission lines than observed, regardless of density and metallicity.

While most of the lines in the models are consistent with the observations, the $[\mathrm{O}$ III $] 4959,5007$ ones are two to three times fainter than observed if we assume that $\mathrm{N} / \mathrm{O}=15$ like in the atmosphere of the star. Moreover, such a high N/O ratio is inconsistent with the ones for other LBVs (Table 7). This inconsistency could be resolved if the oxygen abundance of the nebula were three times greater than the one implied by the CMFGEN model - that is if it is $\mathrm{N} / \mathrm{O}=5$ as we list in Table 7 . The difference between the chemical compositions of the nebula and the atmosphere does not contradict the predictions of evolutionary theory. Indeed, according to the predictions of evolutionary tracks (e.g. Groh et al. 2014) the surface abundance of oxygen rapidly drops by about 1.5 orders of magnitude during the LBV phase, while the nitrogen abundance increases by only two times. At the same time, the nebula may be formed well before the LBV stage, during the transition from the main sequence towards blue supergiants (Lamers et al. 2001), and therefore may have increased nitrogen and normal (equal to surrounding metallicity) oxygen abundance.

The CLOUDY model only partially recovered the broad [Fe III] 5270 presented in the spectrum of GR 290 (Fig. 6). Probably it is related to the adopted geometry of the model that would require future investigations.

Table 7 presents the comparison of GR 290's nebula parameters with those of four other LBVs belonging to our Galaxy, the Milky Way (MW), and the Large Magellanic Cloud (LMC). As we can see the sizes of all five nebulae are similar, and that of GR 290's nebula is in good agreement with the average sizes 



Fig. 8. Upper panel: identification chart for GR290. Dashed-dotted line shows the slit position during July observations. Lower panel: tracing of the two-dimensional spectral image obtained of GR 290 in July 2017 along the spatial direction centred on the $\mathrm{H} \alpha$ emission line. GR 290 is located at zero point and has a strong and sharply-peaked $\mathrm{H} \alpha$ line with asymmetrically extended wings.

of LBVs according to Weis (2012). However, $n_{\mathrm{e}}$ for GR 290's nebula is significantly lower ${ }^{9}$, just like the one for WRA 751 (Garcia-Lario et al. 1998).

Fabrika et al. (2005) reported the marginal detection of a circumstellar nebula around GR 290 based on integral field spectroscopy. They found a radial velocity gradient within the nebula of $44 \pm 11 \mathrm{~km} \mathrm{~s}^{-1}$ and an angular size $\sim 9$ " in the NE-SW direction. The detection is classified as marginal because their spectra did not cover the $\mathrm{H} \alpha$ spectral region and they did not detect the [O III] lines, so the results are based on the analysis of the $\mathrm{H} \beta$ line profiles alone. However, if real, this region would correspond to material lying intermediate between the compact $\mathrm{H}$ II region that we detect $(\mathrm{R} \leq 4 \mathrm{pc})$ in the emission-line spectrum and the material that we are able to resolve at a projected distance of $16-40 \mathrm{pc}$ to the east of GR 290. If this intermediate material reflects an asymmetric ejection, it increases the possi-

\footnotetext{
9 Here we assume that $n_{\mathrm{H}}$ is equivalent to $n_{\mathrm{e}}$.
}

Table 6. Heliocentric radial velocities of nebular lines $\left(\mathrm{km} \mathrm{s}^{-1}\right)$, August spectrum.

\begin{tabular}{lccc}
\hline \hline $\begin{array}{l}\text { Position: } \\
\text { Line }\end{array}$ & $\begin{array}{c}1 \\
\text { GR 290 }\end{array}$ & $\begin{array}{c}2 \\
\text { M 33-back }\end{array}$ & $\begin{array}{c}3 \\
\text { OB89 }\end{array}$ \\
\hline$[S I I] 6716.44$ & $\ldots$ & -208 & -223 \\
{$[S I I] 6730.82$} & $\ldots$ & $\ldots$ & -226 \\
{$[N I I] 6548.01$} & -188 & $-147:$ & $-180:^{a}$ \\
{$[N I I] 6583.45$} & -189 & -233 & -211 \\
$H \alpha 6562.83$ & -179 & -194 & -200 \\
{$[O I I I] 4958.90$} & -206 & $\ldots$ & $\ldots$ \\
{$[O I I I] 5006.84$} & $-201^{b}$ & $\ldots$ & $\ldots$ \\
\hline
\end{tabular}

Notes. ${ }^{(a)}$ The [N II] 6548 A line is significantly weaker than [N II] 6583 and is partially blended with the $H \alpha$ emission line. For this reason, we consider this measurement to have a larger uncertainty in RV than the rest, which is indicated with a colon (:) in the table; ${ }^{(b)}$ [O III] 5007 was measured on the one-dimensional spectrum with a three-Gaussian fit to correct for the presence of the neighbouring He I $5015 \AA$ line whose PCyg absorption component overlaps its red wing.

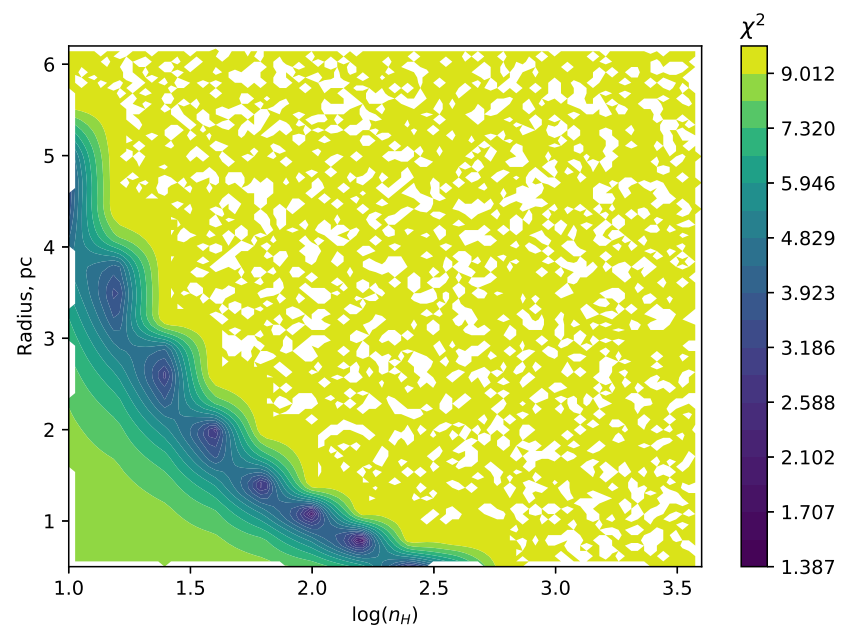

Fig. 9. Chi-square distribution for the CLOUDY models with different densities and outer radii. Chemical composition here is the same as for GR 290 best-fit CMFGEN model, but with two times larger abundance of $\mathrm{O}$ and $\mathrm{Fe}$. For clarity all values of $\chi^{2}>10$ were changed to ten in this plot.

bility that the inner compact $\mathrm{H}$ II region is also asymmetrical. It is also interesting to note that a preliminary CLOUDY photoionization model indicates that GR 290 could be responsible for producing at least a fraction of the observed [S II] at these distances, with no [O III]. However, a more detailed analysis of the extended regions requires observations with higher $\mathrm{S} / \mathrm{N}$ than those we currently present.

\section{Conclusions}

We present results of long-slit observations obtained with GTC in 2016 of the LBV/WR object GR 290 (Romano's star). GR 290 lies in a relatively external region of the M33 galaxy, where metallicity is low compared to solar values. Fitting CMFGEN models to the stellar spectrum we find a current effective temperature of $T_{\text {eff }}=27000-30000 \mathrm{~K}$ at a radius $R_{2 / 3}=27-23 R_{\odot}$. The star's mass-loss rate is $1.5 \times 10^{-5} M_{\odot} \mathrm{yr}^{-1}$. 
Table 7. Nebular parameters for some LBVs and of GR290.

\begin{tabular}{lllllllll}
\hline \hline Name & Galaxy & Type & $\begin{array}{l}\text { Max.size } \\
(\mathrm{pc})\end{array}$ & $\begin{array}{l}n_{\mathrm{e}} \\
\left(\mathrm{cm}^{-3}\right)\end{array}$ & $\mathrm{N} / \mathrm{O}$ & $\mathrm{N} / \mathrm{S}$ & $(\mathrm{N}+\mathrm{O}) / \mathrm{H}$ & Ref \\
\hline AG Car & MW & Bipolar & $1.4 \times 2$ & $820 \pm 170$ & $6 \pm 2$ & $<45$ & $4 \times 10^{-4}$ & 1,2 \\
P Cyg & MW & Spherical & $0.2 / 0.84$ & 600 & $>0.16$ & 33 & $0.4-3 \times 10^{-3}$ & 1,2 \\
WRA 751 & MW & Spherical/bipolar & 0.6 & 200 & $3_{-1.5}^{+3}$ & & $4-4.9 \times 10^{-4}$ & 1,3 \\
R 127 & LMC & Bipolar & 1.3 & $720 \pm 90$ & $0.9 \pm 0.4$ & $<34$ & $3.2 \times 10^{-4}$ & 1,2 \\
S 119 & LMC & Spherical/outflow & 1.8 & $680 \pm 170$ & $1.9 \pm 0.5$ & $<78$ & $3.8 \times 10^{-4}$ & 1,2 \\
& & & & & & & & \\
GR 290 & M33 & & $0.8-4$ & $160^{a}$ & $5^{b}$ & 65 & $7.3 \times 10^{-4}$ & This work \\
\hline
\end{tabular}

Notes. Types and sizes are taken from (1) Weis (2011) while $n_{\mathrm{e}}$ and chemical composition are taken from (2) Lamers et al. (2001) and (3) Garcia-Lario et al. (1998). ${ }^{(a)}$ Here we show CLOUDY-estimated $n_{\mathrm{H}}$ as $n_{\mathrm{e}} \cdot{ }^{(b)}$ The text provides a discussion on how we estimated this quantity.

The terminal wind speed $v_{\infty}=620 \mathrm{~km} \mathrm{~s}^{-1}$ is faster than ever before recorded, while the current luminosity $L_{*}=$ (3.1-3.7) $\times 10^{5} L_{\odot}$ is the lowest ever deduced. The remaining parameters of the 2016 observation fall within the ranges of values obtained at previous epochs as described in Polcaro et al. $(2016)^{10}$. We also confirm previous conclusions that the stellar wind is overabundant in $\mathrm{He}$ and $\mathrm{N}$ and underabundant in $\mathrm{C}$ and $\mathrm{O}$.

We find the star in GR 290 to be surrounded by an unresolved compact $\mathrm{H}_{\mathrm{II}}$ region with dimensions $\leq 4 \mathrm{pc}$, and with chemical abundances that are consistent with those derived from the stellar wind lines. Therefore, this compact $\mathrm{H}$ II region appears to be largely composed of material ejected from the star. In addition, we find partially resolved emission from a more extended ISM region, which appears to be asymmetrical, with a larger extent to the east (16-40 pc) than to the west. If associated with GR 290, this more extended structure would suggest that GR 290 has undergone significant mass loss over the past $10^{4}-10^{5} \mathrm{yr}$.

The luminosity and effective temperature of GR 290 allow us to place it on the HRD and estimate its mass. Using the Brott et al. (2011) non-rotating evolutionary grid, $M \sim 40-$ $50 M_{\odot}$, while with the Chieffi \& Limongi (2013) tracks its mass is $\sim 60 M_{\odot}$. The enhanced He and $\mathrm{N}$ chemical abundances, along with deficiencies in $\mathrm{C}$ and $\mathrm{O}$ derived from the CMFGEN models, indicate that the surface layers of GR 290 contain significant amounts of nuclear processed material, such as would be expected for a star that has left the main sequence and is becoming a WR star (Langer et al. 1994). Adding this to the recent LBV-like eruptive behaviour and to the presence of a compact $\mathrm{H}$ II region that also has altered chemical abundances with respect to typical H II regions, one is led to conclude that GR 290 is in an advanced stage of evolution, either nearing the end of its core H-burning phase or in later phases.

There are now a number of reported supernova (SNIIn) events that have been observed in extragalactic regions where there has been a massive ejection of material prior to the $\mathrm{SN}$ event - often within ten years - and which is believed to have arisen in an LBV (Foley et al. 2007; Smith et al. 2007, 2011; Smith 2007; Pastorello et al. 2008; Dessart et al. 2011). Thus, given the apparent late evolutionary state of GR 290, it is a good candidate to be a progenitor of a similar event sometime in the future.

\footnotetext{
${ }^{10}$ The larger $T_{\text {eff }}$ derived by Polcaro et al. (2016) for GR290 during the 2008 and 2014 minima can be attributed to the fact that the stellar parameters have been computed adopting $\beta=1$.
}

Acknowledgements. We dedicate this publication to the memory of our dear colleague Vito Francesco Polcaro, who passed away shortly before the final acceptance of the paper. Dr. Vito Francesco Polcaro passed away on February 11, 2018 in Roma. Besides GR 290, Dr. Polcaro prompted many relevant investigations of hot luminous stars, X-ray counterparts, and circumstellar nebulae, best exploiting in particular the performances of medium and small telescopes. $\mathrm{He}$ inspired amateurs to participate in the photometric monitoring of topical objects. During his last years Dr. Polcaro was very active in the field of archeoastronomy, and organized campaigns in paleolithic sites and conferences gathering astronomers and archeologists.

We would like to thank the anonymous referee for a helpful and insightful report. We thank the GTC observatory staff for obtaining the spectra and Antonio Cabrera-Lavers for guidance in processing the observations. GK acknowledges support from CONACYT grant 252499 and thanks Ulises Amaya for computer support. OM acknowledges Russian Foundation for Basic Research grant 16-0200148 and AV ČR project RVO:67985815. OE acknowledges Russian Foundation for Basic Research grant 18-02-00976.

\section{References}

Abolmasov, P. 2011, New Astron., 16, 421

Afanasiev, V. L., \& Moiseev, A. V. 2005, Astron. Lett., 31, 194

Bresolin, F. 2011, ApJ, 730, 129

Brott, I., de Mink, S. E., Cantiello, M., et al. 2011, A\&A, 530, A115

Chieffi, A., \& Limongi, M. 2013, ApJ, 764, 21

Clark, J. S., Castro, N., Garcia, M., et al. 2012, A\&A, 541, A146

Conti, P. S. 1984, in Observational Tests of the Stellar Evolution Theory, eds. A. Maeder, \& A. Renzini, IAU Symp., 105, 233

Dessart, L., Hillier, D. J., Livne, E., et al. 2011, MNRAS, 414, 2985

Ederoclite, A., \& Cepa, J. 2010, ASP Conf. Ser., 434, 253

Ekström, S., Georgy, C., Eggenberger, P., et al. 2012, A\&A, 537, A146

Fabrika, S., Sholukhova, O., Becker, T., et al. 2005, A\&A, 437, 217

Ferland, G. J., Korista, K. T., Verner, D. A., et al. 1998, PASP, 110, 761

Ferland, G. J., Chatzikos, M., Guzmán, F., et al. 2017, Rev. Mex. Astron. Astrofis., 53, 385

Foley, R. J., Smith, N., Ganeshalingam, M., et al. 2007, ApJ, 657, L105

Galleti, S., Bellazzini, M., \& Ferraro, F. R. 2004, A\&A, 423, 925

Garcia-Lario, P., Riera, A., \& Manchado, A. 1998, A\&A, 334, 1007

Georgiev, L., Koenigsberger, G., Hillier, D. J., et al. 2011, AJ, 142, 191

Gräfener, G., Owocki, S. P., \& Vink, J. S. 2012, A\&A, 538, A40

Groh, J. H., Hillier, D. J., Damineli, A., et al. 2009, ApJ, 698, 1698

Groh, J. H., Meynet, G., \& Ekström, S. 2013a, A\&A, 550, L7

Groh, J. H., Meynet, G., Georgy, C., \& Ekström, S. 2013b, A\&A, 558, A131

Groh, J. H., Meynet, G., Ekström, S., \& Georgy, C. 2014, A\&A, 564, A30

Hillier, D. J., \& Miller, D. L. 1998, ApJ, 496, 407

Hillier, D. J., \& Miller, D. L. 1999, ApJ, 519, 354

Humphreys, R. M., \& Davidson, K. 1994, PASP, 106, 1025

Humphreys, R. M., \& Sandage, A. 1980, ApJS, 44, 319

Humphreys, R. M., Weis, K., Davidson, K., Bomans, D. J., \& Burggraf, B. 2014, ApJ, 790, 48

Humphreys, R. M., Weis, K., Davidson, K., \& Gordon, M. S. 2016, ApJ, 825, 64

Ivanov, G. R., Freedman, W. L., \& Madore, B. F. 1993, ApJS, 89, 85

Koenigsberger, G., Morrell, N., Hillier, D. J., et al. 2014, AJ, 148, 62

Kurtev, R., Sholukhova, O., Borissova, J., \& Georgiev, L. 2001, Rev. Mex. Astron. Astrofis., 37, 57 
Lamers, H. J. G. L. M., \& Cassinelli, J. P. 1999, Introduction to Stellar Winds (Cambridge: Cambridge University Press), 452

Lamers, H. J. G. L. M., Nota, A., Panagia, N., Smith, L. J., \& Langer, N. 2001, ApJ, 551, 764

Langer, N., Hamann, W.-R., Lennon, M., et al. 1994, A\&A, 290, 819

Magrini, L., Stanghellini, L., Corbelli, E., Galli, D., \& Villaver, E. 2010, A\&A, 512, A63

Martayan, C., Lobel, A., Baade, D., et al. 2016, A\&A, 587, A115

Maryeva, O., \& Abolmasov, P. 2010, Rev. Mex. Astron. Astrofis., 46, 279

Maryeva, O., \& Abolmasov, P. 2012, MNRAS, 419, 1455

Massey, P., Armandroff, T. E., Pyke, R., Patel, K., \& Wilson, C. D. 1995, AJ, 110,2715

Meynet, G., \& Maeder, A. 2005, A\&A, 429, 581

Meynet, G., Georgy, C., Hirschi, R., et al. 2011, Bull. Soc. Roy. Sci. Liège, 80, 266

Pastorello, A., Quimby, R. M., Smartt, S. J., et al. 2008, MNRAS, 389, 131

Polcaro, V. F., Gualandi, R., Norci, L., Rossi, C., \& Viotti, R. F. 2003, A\&A, 411, 193

Polcaro, V. F., Rossi, C., Viotti, R. F., et al. 2011, AJ, 141, 18

Polcaro, V. F., Maryeva, O., Nesci, R., et al. 2016, AJ, 151, 149

Puls, J., Kudritzki, R.-P., Herrero, A., et al. 1996, A\&A, 305, 171

Romano, G. 1978, A\&A, 67, 291

Rosolowsky, E., \& Simon, J. D. 2008, ApJ, 675, 1213

Sander, A. A. C., Hamann, W.-R., Todt, H., Hainich, R., \& Shenar, T. 2017, A\&A, 603, A86

Sholukhova, O. N., Fabrika, S. N., Vlasyuk, V. V., \& Burenkov, A. N. 1997, Astron. Lett., 23, 458

Sholukhova, O., Zharova, A., Fabrika, S., \& Malinovskii, D. 2002, in IAU Colloq. 185: Radial and Nonradial Pulsationsn as Probes of Stellar Physics, eds. C. Aerts, T. R. Bedding, \& J. Christensen-Dalsgaard, ASP Conf. Ser., 259,522

Sholukhova, O. N., Fabrika, S. N., Zharova, A. V., Valeev, A. F., \& Goranskij, V. P. 2011, Astrophys. Bull., 66, 123

Smith, L. F. 1968, MNRAS, 138, 109

Smith, L. F., Shara, M. M., \& Moffat, A. F. J. 1996, MNRAS, 281, 163

Smith, N. 2007, in Supernova 1987A: 20 Years After: Supernovae and GammaRay Bursters, eds. S. Immler, K. Weiler, \& R. McCray, AIP Conf. Ser., 937 163

Smith, N., Li, W., Foley, R. J., et al. 2007, ApJ, 666, 1116

Smith, N., Li, W., Filippenko, A. V., \& Chornock, R. 2011, MNRAS, 412, 1522 Szeifert, T. 1996, in Liège Int. Astrophys. Colloq., eds. J. M. Vreux, A. Detal, D. Fraipont-Caro, E. Gosset, \& G. Rauw, 33, 459

van der Hucht, K. A., Conti, P. S., Lundstrom, I., \& Stenholm, B. 1981, Space Sci. Rev., 28, 227

Vink, J. S. 2012, in Eta Carinae and the Supernova Impostors, eds. K. Davidson, \& R. M. Humphreys, Astrophys. Space Sci. Lib., 384, 221

Viotti, R. F., Rossi, C., Polcaro, V. F., et al. 2006, A\&A, 458, 225

Viotti, R. F., Galleti, S., Gualandi, R., et al. 2007, A\&A, 464, L53

Walborn, N. R., Gamen, R. C., Morrell, N. I., et al. 2017, AJ, 154, 15

Weis, K. 2011, in Active OB Stars: Structure, Evolution, Mass Loss, and Critical Limits, eds. C. Neiner, G. Wade, G. Meynet, \& G. Peters, IAU Symp., 272, 372

Weis, K. 2012, in Proceedings of a Scientific Meeting in Honor of Anthony F. J Moffat, eds. L. Drissen, C. Robert, N. St-Louis, \& A. F. J. Moffat, ASP Conf. Ser., 465, 213

Wolf, B. 1989, A\&A, 217, 87

Zharova, A., Goranskij, V., Sholukhova, O. N., \& Fabrika, S. N. 2011, Peremennye Zvezdy Prilozhenie, 11, 11 